\title{
Phase variation in Salmonella: analysis of Hin recombinase and hix recombination site interaction in vivo
}

\author{
Kelly T. Hughes, ${ }^{1}$ Philip Youderian, ${ }^{2}$ and Melvin I. Simon ${ }^{1,3}$ \\ Division of Biology 147-75, California Institute of Technology, Pasadena, California 91125 USA $_{i}{ }^{2}$ Department of Biological \\ Sciences, University of Southern California, Los Angeles, California 90089 USA
}

\begin{abstract}
The bacteriophage P22-based challenge phase selection was used to characterize the binding of Salmonella Hin recombinase to the wild-type hixL and hix $R$ recombination sites, as well as to mutant and synthetic hix sequences in vivo. Hin recombinase binds to the hixL or hixR recombination sites and represses transcription from an upstream promoter in the challenge phage system. Hin-mediated repression results from Hin associating into multimers either prior to binding or during the binding process at the hix operator sites (cooperativity). The ability of Hin multimers to repress transcription is eliminated when the hix 13-bp half-sites are rotated to opposite sides of the DNA helix by inserting 4 bp between them. Insertion of 1 bp between halfsites reduces overall repression. Hin also binds one of the hixL half-sites to repress transcription, but only when high levels of Hin protein are present in the cell. Mutations have been identified in the hix sites that impair Hin binding. Five of the 26 bp in the hix sites are critical; sites with base-pair substitutions at these five positions show greatly reduced binding. Three additional base pairs make minor contributions to binding. These results are consistent with the results of binding studies between Hin and the hix sites in vitro.
\end{abstract}

[Key Words: Hin recombinase; DNA binding; phage P22]

Received January 28, 1988; revised version accepted June 2, 1988.

Salmonella can express each of two different flagellar antigens, $\mathrm{Hl}$ and $\mathrm{H} 2$, alternatively (Stocker 1949; lino 1969). The mechanism that mediates flagella phase variation in Salmonella is the reversible inversion of a 996bp segment of the chromosome (Fig. 1) (Zieg et al. 1977, 1978; Zieg and Simon 1980). In one orientation, this inversion segment places a promoter upstream of the $H 2$ flagellin and the $r H 1$ (repressor of $\mathrm{H1}$ ) genes, resulting in the production of $\mathrm{H} 2$ flagella and the $\mathrm{rH} 1$ repressor protein. The $\mathrm{rHl}$ repressor acts in trans to repress transcription of the $H 1$ flagellin gene, which maps at a different location on the Salmonella chromosome (Iino 1977; Silverman et al. 1979). Inversion of the DNA segment to the other orientation positions the promoter so that neither $\mathrm{H} 2$ nor $\mathrm{rH} 1$ are transcribed, and only $\mathrm{H} 1$ flagellin is produced. Thus, the inversion mechanism is an on/off switch for the two antigenically different Salmonella flagellin proteins.

The mechanism of phase variation has been characterized extensively at the molecular and biochemical level. Inversion of the 996-bp segment is the result of a sitespecific recombination event between two partially homologous 26-bp recombination sites, hix $L$ and hix R (Fig. 2 ), that flank the left and right ends of the invertible segment, respectively (Johnson and Simon 1985). The re-

${ }^{3}$ Corresponding author. combination reaction is mediated by the Hin recombinase, encoded by a gene (hin) within the invertible segment (Silverman and Simon 1980; Johnson et al. 1986). In addition to Hin protein and the hix $L$ and hixR sites, the inversion reaction requires a DNA recombinational enhancer site and two other cell proteins, Fis and HU (Johnson and Simon 1985; Johnson et al. 1986). The Fis protein interacts directly with the enhancer, whereas the HU protein is thought to contribute to the efficiency of the recombination reaction by enhanced bending of the DNA (Bruist et al. 1987a; Johnson et al. 1987). All of these factors orchestrate the formation of a specific protein-DNA complex necessary for the breakage and rejoining of DNA at the hixL and hixR sites resulting in inversion (Bruist et al. 1987b).

Three other site-specific inversion mechanisms similar to the Hin system have been found in phages $\mathrm{Mu}$ and $\mathrm{P} 1$, and in the Escherichia coli defective prophage el4 (Plasterk et al. 1983). The Mu, P1, e14, and Hin systems share recombination site and recombinase protein sequence homology (Kutsukake and lino 1980; Szekeley and Simon 1983). The Mu and P1 systems also require Fis and a recombinational enhancer and are thought to use a mechanism for site-specific inversion similar to the Hin system (Huber et al. 1985; Kahman et al. 1985, 1987).

In this paper, we describe the initial genetic characterization of the specific protein-DNA interactions be- 
Hughes et al.
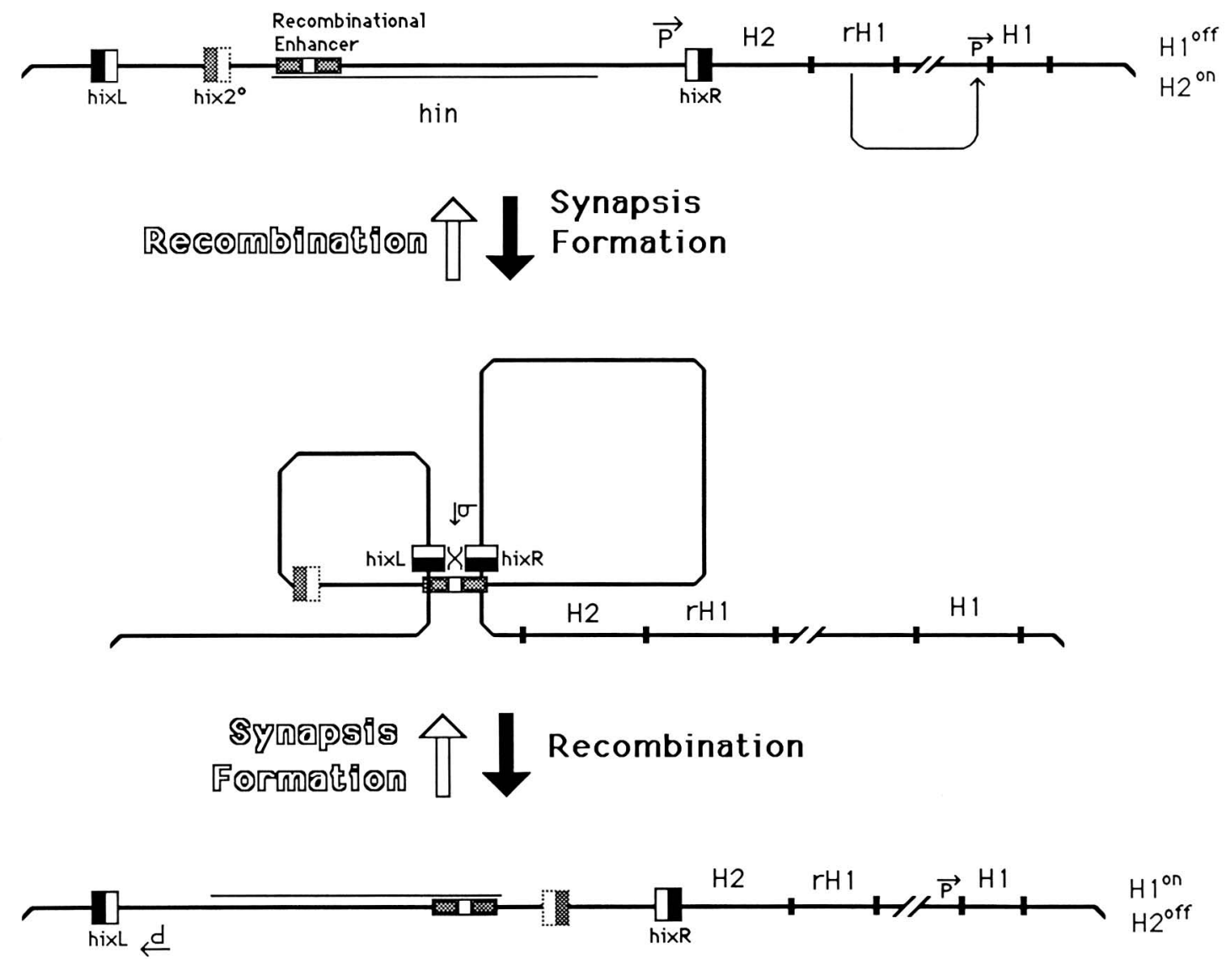

Figure 1. Hin-mediated site-specific recombination. The reversible, Hin-mediated recombination mechanism that switches Salmonella flagellin production between the $\mathrm{H} 1$ and $\mathrm{H} 2$ flagellin antigens is shown. (Top) The $\mathrm{H} 2$ promoter provides a transcription initiation site for transcription and subsequent translation of the $H 2$ and $r H 1$ genes. The $r H 1$ gene encodes a protein that acts to repress transcription from the $H 1$ promoter. Thus, only the $\mathrm{H} 2$ flagellin is produced in the top orientation. Hin mediates a site-specific recombination reaction between the hix $L$ and hix $R$ recombination sites. The reaction also requires a host protein, Fis, which interacts directly with the recombinational enhancer sequence and probably the Hin protein as well to form a synaptonemal-like complex necessary for recombination to occur. The recombinational event inverts the segment of the chromosome between the hix sites. (Bottom) There is no promoter to express the $H 2$ and $r H 1$ genes, and only the Hl flagellin is produced.

tween Hin recombinase and the hix recombination sites in vivo. We have used the bacteriophage P22 challenge phage selection, which is designed to assay the relative strengths of protein-DNA interactions in vivo (Benson et al. 1986). In this system, Hin binding to a hix site is assayed as a repressor binding to its operator site. We have replaced the normal operator for the P22 ant gene (Mnt binding site) with various hix sites that may act as alternate operator sequences for controlling ant gene expression (Fig. 3). When Hin is absent or cannot bind a given hix operator site, the ant gene is derepressed, and its product, antirepressor, is produced constitutively.

$1312111098776544321+4-2 \cdot 3-4-5$-6 -7 -8 -9-10-11-12-13

hixL: 5'-TTCTTGAAAACCAAGGT TTTTGATAA-3' 3'-AAGAAC T T T TGGT T CCAAAAAC TAT T-5'

hixR: 5'-T TATCAAAAACC T TCCAAAAGGAAAA-3' 3'-AATAGT T T T TGGAAGGT T T TCC T T T T-5'

Figure 2. The sequence of the hixL, hixR, and hixS sites as they are read counterclockwise on the Salmonella linkage map.
hixS: 5'-TCT TCCT TATCTGATGTAAAGGAGAA-3' 


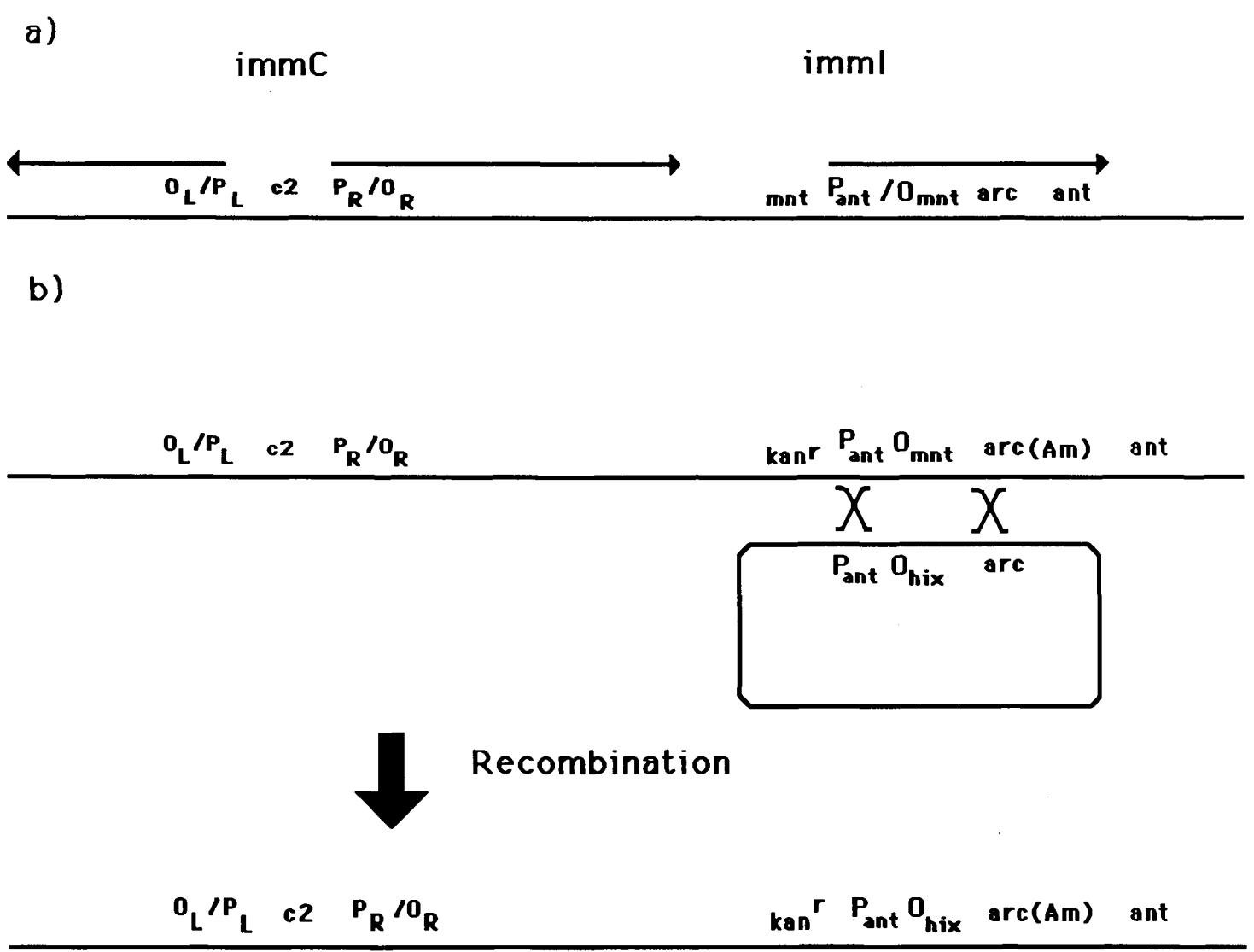

Figure 3. Construction of the P22 hix challenge phages. $(a)$ A diagram of the wild-type phage P22 showing the immunity regions. $(b)$ A recombination event between P22 phage $[K n 9 \operatorname{arcH}(\mathrm{am})]$ and the pPY190 plasmid with a hix sequence cloned into the $\mathrm{O}_{m n t}$ site. This results in a challenge phage in which transcription from $\mathrm{P}_{\text {ant }}$ is regulated by Hin, which can bind to the $\mathrm{O}_{\text {hix }}$ site and repress transcription.

Antirepressor inhibits the activity of P22 c2 repressor (analogous in function with phage $\lambda c I$ repressor), prevents the establishment of lysogeny, and channels the superinfecting phage into lytic development (Susskind and Youderian 1983). When Hin is present and binds a given hix site, transcription of the ant gene is repressed, and the challenge phage carrying the hix site can lysogenize the Salmonella host.

The recovery of lysogens is by direct selection because the challenge phage carries a kanamycin resistance $\left(\mathrm{Kan}^{\mathrm{r}}\right.$ ) determinant (Youderian et al. 1983). The relative binding efficiency of Hin protein to a particular site is measured as the frequency of lysogeny of a challenge phage with this site, a value that ranges over eight orders of magnitude. In this assay, the level of Hin can be varied, because Hin is expressed from the tac promoter, which is under the control of Lac repressor. The level of Lac repressor and, therefore, hin transcription can be increased or decreased by increasing or decreasing the level of the inducer of Lac repressor, isopropyl $\beta$-D-thiogalactopyranoside (IPTG). The relative binding efficiencies of Hin to different hix sites can thus be inferred by assaying the frequency of lysogeny of challenge phages with different hix sites at varying IPTG concentrations.

\section{Results}

Use of the P22 challenge phage selection to study Hin binding to hix recombination sites in vivo

To study binding of Hin recombinase to various hix sites in vivo, the bacteriophage $\mathrm{P} 22$ challenge phage selection was used (Benson et al. 1986). This selection is based on the genetic properties of the P22 immI region (Fig. 3) (Susskind and Youderian 1983). The ant gene product, antirepressor, inhibits the activity of the primary P22 repressor, $c 2$. The ant gene, in turn, is regulated by the Mnt repressor, which binds a single, symmetric site the ant operator region) located at the startpoint of transcription of the ant gene, preventing transcription of the ant gene. If ant is not repressed, P22 cannot lysogenize its Salmonella host.

By constructing a P22 phage that has a Kan ${ }^{\mathrm{r}}$ gene in place of the mnt structural gene, lysogens can be selected and the frequency of lysogenization readily measured. The ant operator region has been replaced by a multiple cloning site for inserting synthetic operators at the ant gene transcriptional startpoint. Any DNA segment whose in vivo binding to a particular protein is to be characterized is inserted at the ant operator site. When a protein can bind the artificial operator at the ant 
operator region, ant is repressed and lysogenization frequencies are determined by measuring the frequencies of $\mathrm{Kan}^{\mathrm{r}}$ survivors. This system has been applied successfully to study in vivo binding of the $E$. coli $\mathrm{Lac}, \mathrm{Gal}$, and Trp repressors, phage $\lambda c I$ and Cro repressors, and the site-specific recombinase FLP protein of yeast Saccharomyces cerevisiae to their respective DNA-binding sites (Benson et al. 1986; Bass et al. 1987; Lebreton et al. 1988).

We have constructed a set of hix challenge phages, including phages with wild-type hix sequences and phages with mutant sequences. The wild-type sequences are hix L, hixR, and a secondary Hin-binding site (hixS) located upstream of the hin translation initiation region in Salmonella and are shown in Figure 2. The hixS site binds Hin weakly in vitro and may be part of an autogenous regulatory mechanism (Bruist et al. 1987b). The hixS site is unrelated to the enhancer site that binds the Fis protein and is necessary for the inversion reaction. Challenge phages were constructed that placed the hixL, hixR, and hixS sites into the operator locus for the P22 ant gene. We found that none of these phages is able to lysogenize Salmonella typhimurium strains $\left(\leqslant 10^{-8}\right)$, unless they carry Hin-producing plasmids. This suggests that the level of Hin produced from the chromosome is not sufficient to repress these challenge phages.

The challenge phage assays were all performed on strain MS1868, carrying the pKH66 plasmid that harbors both the $1 a c I^{\mathrm{Q}}$ gene and the hin gene expressed from the tac promoter. Hin expression in cells to be infected with challenge phage was induced by the addition of IPTG to the growth medium prior to infection. The levels of Hin were varied by varying the amounts of IPTG, and the frequencies of $\operatorname{Kan}^{\mathrm{x}}$ survivors at each different IPTG concentration were measured. As shown in Figure 4, when MS1868 (pKH66) is grown at low IPTG concentrations, only a small fraction of cells survive infection by challenge with either the hix $L$ or hixR sites. As the IPTG concentration is increased in small increments between $6 \times 10^{-5}$ and $6 \times 10^{-4} \mathrm{M}$, the frequency of lysogeny of these challenge phages increases dramatically, from $10^{-4}$ to $>10 \%$. The increase in the frequency of lysogeny responds sigmoidally to an increase in IPTG concentration and, therefore, the level of Hin. It was found (see below) that the concentration of Hin in the cell also increases sigmoidally over this range of IPTG concentrations, but the total increase is only threefold.

The precise response to challenge phage depends on the nature of the hix sites and also on the disposition of the site with respect to the ant promoter. Similar lysogenization curves are obtained for challenge phages with the hix $L$ sequence in either orientation. In contrast, the $h i x R$ challenge phages show different dose-response curves for each of the two orientations of hixR. One of the hix $R$ challenge phages shows a biphasic curve (Fig. 4) demonstrating orientation dependence of the challenge phage selection. When the challenge phage selection was performed with the hixS site, no lysogeny was detected in either orientation. This result suggests (but does not rule out) that Hin does not bind appreciably to the hixS

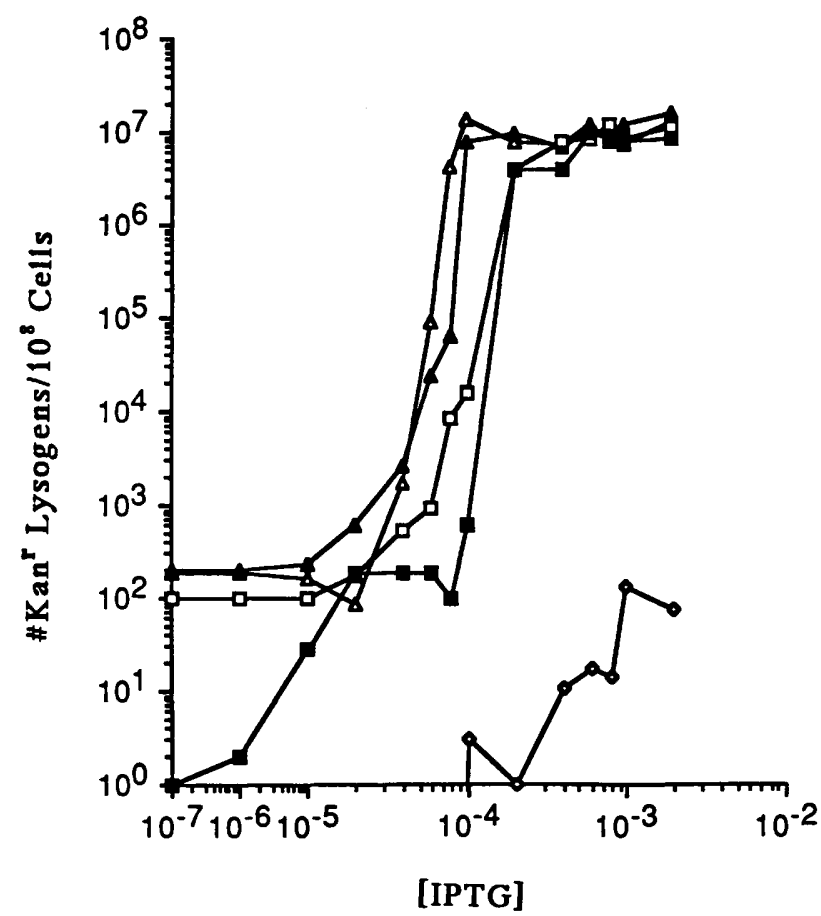

Figure 4. Challenge phage assays with the hixL [orientations I $(\Delta)$ and II $(\boldsymbol{\Delta})]$, hixR [I ( $)$ and II ( $\square)]$, and half-site [I $(\diamond)$ and II] challenge phages. These curves represent the frequency of Kan ${ }^{r}$ lysogens as a function of increasing amounts of IPTG. The recipient in all assays is MS1868(pKH66). In these assays, Hin is expressed from the tac promoter in plasmid pKH66 whose transcription is regulated by the $\mathrm{LacI}^{\mathrm{Q}}$ repressor also encoded in pKH66. The levels of Hin are increased by increasing levels of Lac inducer, IPTG. The curves show relative binding efficiencies of Hin to the hix sites in the different challenge phages tested. Of the six half-site challenge phages tested, only the $1 / 2$ hix $C$ (orientation I, see Fig. 6) was able to lysogenize the recipient cells.

site, and this site probably plays little role in Hin autoregulation. These results demonstrate that the challenge phage assay can be used to characterize binding of Hin to the hix $L$ and hix $R$ recombination sites in vivo.

\section{Determination of in vivo Hin concentration as a function of increasing IPTG levels}

To confirm that small increases in IPTG concentrations correlate directly to small increases in Hin levels, the levels of Hin protein produced in vivo in strain MS1868 (pKH66) induced with the IPTG concentrations used in the challenge phage selections were determined by Western blot analysis. As shown in Figure 5A, small increases in IPTG concentrations result in gradual increases in Hin levels. By scanning the Hin bands in Figure 5A with a densitometer (LKB 2022 Ultrascan Laser Densitometer) and comparing the densities to those for purified Hin standards, the number of Hin molecules per cell was found to increase from $\sim 7000$ molecules per cell at an IPTG concentration of $2 \times 10^{-5} \mathrm{M}$ to 

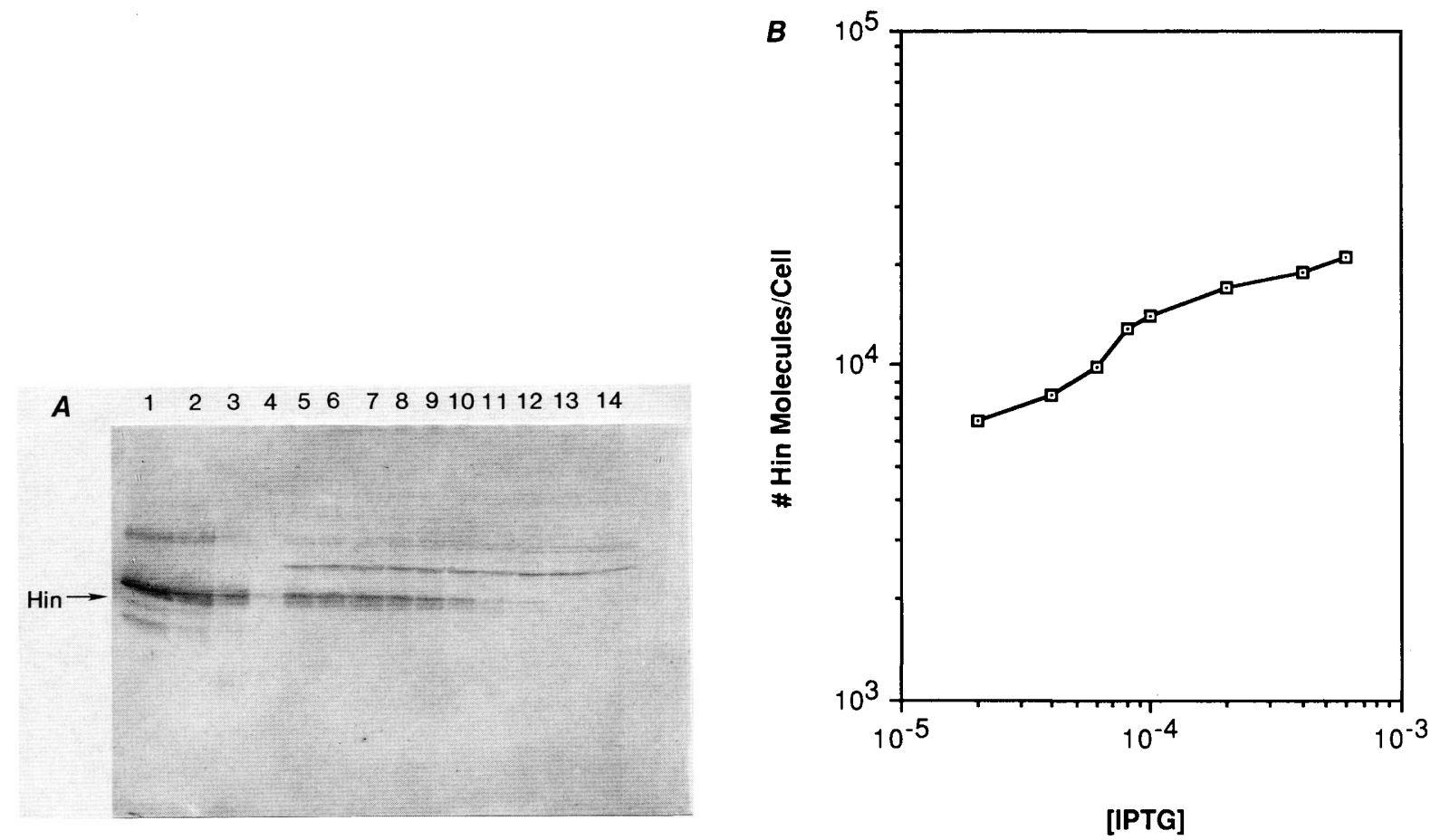

Figure 5. (A) Variable Hin induction by IPTG. Strain MS1868 carrying plasmid pKH66 was grown in the presence of varying IPTG levels (see Materials and methods), and the levels of Hin produced were assayed by antibody staining. Lanes 1-4 contain 500, 250, 100, and $50 \mathrm{ng}$ of purified Hin protein, respectively. Lanes 5-12 contain extracts of MS1868 carrying pKH66 induced with $6 \times 10^{-4}$, $4 \times 10^{-4}, 2 \times 10^{-4}, 8 \times 10^{-4}, 8 \times 10^{-5}, 6 \times 10^{-5}, 4 \times 10^{-5}$, and $2 \times 10^{-5} \mathrm{M}$ IPTG, respectively: Lane 13 contains uninduced cells and lane 14 contains MS1868 without plasmid. There is an additional band picked up by the antibody, which migrates slower than Hin. This band is present in both $E$. coli and $S$. typhimurium and its presence is independent of the presence or absence of Hin protein in the cell (Bruist and Simon 1984). (B) The number of Hin molecules per cell under different IPTG-inducing concentrations. The Hin bands in the Western blots in $A$ and other similar blots (data not shown) were scanned with a densitometer, and these densities were compared to those of purified Hin standards to estimate the number of Hin molecules per cell in the presence of different concentrations of IPTG inducer.

21,000 molecules per cell at an IPTG concentration of $6 \times 10^{-4} \mathrm{M}$. Although Hin levels increase only threefold over this range of IPTG concentrations, the increase is sigmoidal (Fig. 5B).

\section{Challenge phage assays on hix half-sites}

The above result that repression at the hixR operator shows a difference depending on orientation suggests that there may be differential binding to each of the half-sites in vivo, as has been shown in vitro (Bruist et al. 1987b). Challenge phages were constructed with each of the three different hix half-sites from hixL and hix $R$ (Figs. 2 and 6). The half-sites in hix L and hix $R$ that are inverted during the recombination reaction are identical. The two half-sites that are not inverted differ from the common, inverted half-site and from one another. Nine out of $13 \mathrm{bp}$ in the hixL half-sites, and 8 out of 13 $\mathrm{bp}$ in the hix $R$ half-sites are symmetrically related.

When the challenge phage assay was performed on each of the three different half-sites in both possible orientations (Fig. 6), we observed that only one of the sites in one orientation showed significant binding to Hin (Fig. 4). The lysogeny resulting from Hin binding to the common half-site, as shown in Figure 4, is at a low frequency compared to Hin binding to the hix full sites, and the increase in the lysogenization frequency with increases in IPTG levels is small compared to the that with full sites. Furthermore, the half-site does not show the sigmoidal increases in lysogenization with small increases in IPTG levels, as was observed using full sites. Thus, Hin is unlike most characterized repressors because it is able to recognize and bind to a half-site.

\section{The binding of Hin to mutant hix operator sequences}

All of the above results are consistent with the hypothesis that at least two molecules of Hin bind the natural hix sites in a cooperative manner. We tested the possible cooperativity of Hin binding by assaying the ability of Hin to repress transcription in mutant challenge phages in which the hix $L$ half-sites have been separated and rotated with respect to one another by small insertions of 1 or $4 \mathrm{bp}$ between the half-sites. A mutant hix $L$ site with one extra base at the dyad (hix $L+1)$ was isolated as a spontaneous mutant of a hix $L$ challenge phage defective in binding Hin (see below). The hixL 
Figure 6. Orientation of the hix sequences in the different challenge phages with respect to the ant gene promoter. For hixL and hixR, orientation $\mathrm{I}$ is the orientation read $5^{\prime}-3^{\prime}$ clockwise on the Salmonella chromosome, as presented in the standard linkage map (Sanderson and Roth 1983); orientation II is the orientation read $5^{\prime}-3^{\prime}$ counterclockwise on the Salmonella chromosome. The $1 / 2$ hix C (I and II) sequences represent the half-site common to both hix $L$ and hixR in each orientation. The $1 / 2$ hix $L$ and $1 / 2$ hix $R$ sequences represent the half-sites unique to hix $L$ and hixR, respectively.

\author{
hixL(I) $5^{\circ}-\mathrm{P}_{\text {ant }}$-TTATCAAAAACCTTGGTTTTCAAGAA-arc-ant \\ hixL(II)5'-P $5_{\text {ant }}$-TTCTTGAAAACCAAGGTTTTTGATAA-arc-ant \\ hixR(1) 5'-P ant - TTTTCCTTTTGGAAGGTTTTTGATAA-arc-ont \\ hixR(II) 5'-P $5_{\text {snt }}$-TTATCAAAAACCTTCCAAAAGGAAAA-orc-ont \\ $1 / 2$ hixC(1) 5'-P ant - TTATCAAAAACCA-arc-ant \\ $1 / 2$ hixC(II) 5'-P ant -TGGTTTTTGATAA-arc-ant \\ $1 / 2$ hixL(1) 5'-P ant - TTCTTGAAAACCA-grc-ant \\ $1 / 2$ hixL(II) $5^{\circ}-P_{\text {ant }}-$ TGGTTTTCAAGAA-arc-ant \\ 1/2 hixR(I) 5'-Pant - TTTTCCTTTTGGA-arc-ant \\ $1 / 2$ hixR(II) 5'-Pant -TCCAAAAGGAAAA-arc-ant \\ hixL(1)+1 5'-P ant -TTATCAAAAACCTTTGGTTTTCAAGAA-arc-ant \\ hixC $5^{\prime}-P_{\text {ant }}$-TTATCAAAAACCATGGTTTTTGATAA-arc-ant
}

mutant with four extra bases between the half-sites (hix $L+4$ ) was constructed from a plasmid with a hix $L$ site in which one of the central $A: T$ base pairs is changed to a $\mathrm{T}$ : A base pair, creating an $N$ col restriction site. Plasmid DNA with the hix $L-N c o$ I site was cut with $N c o$, filled in, and religated to construct a plasmid with the hix $L+4$ sequence.

The results of infections with challenge phages carrying the hix $L-N c o I$, hix $L+1$, and hix $L+4$ sites are shown in Figure 7. The frequency of lysogeny of Hinproducing cells infected with the hix $L-N c o$ I challenge phage responds to increasing IPTG concentrations in a way indistinguishable from cells infected with a challenge phage carrying the wild-type hix L site (Figs. 4 and 7). Cells infected with the hixL +1 phage show up to 20 -fold reduced frequencies of lysogeny, depending on the IPTG concentration. The hix $L+1$ phage still shows a sigmoidal binding curve, and the half-maximal frequency of lysogenization occurs at the same IPTG concentration as the half-maximal frequency for hix L. Only a very low level of lysogeny was detected with the hix $L+4$ site at the highest IPTG concentrations.

These results are a direct consequence of the ability of Hin to bind the mutant and wild-type hix operator sites and are not due to the effects of cloned operator sequences on the intrinsic activity of the ant promoter. To demonstrate this, we constructed fusions of the lac operon to the ant promoter with each of the different hix wild-type and mutant sites at the operator locus on single-copy P22 prophages (Fig. 8), and measured the amount of $\beta$-galactosidase made from each hybrid operon. As shown in Table 1 , all fusions direct the synthesis of similar, high levels of $\beta$-galactosidase.
Construction and properties of a perfectly symmetric hix site

We have shown that one of the three different half-sites in hix $L$ and hix $R$ apparently binds Hin more tightly than the other two do. The ability of one of the hix half-sites to bind Hin more tightly than the others suggested that a perfectly symmetric hix site with a pair of the tightest binding half-site, hix $C$, might act as a better operator site than either hixL or hixR. Therefore, we synthesized hix $C$ and inserted this site into the challenge phage ant operator locus. As shown in Figure 7, we found that the hix $C$ sequence is a slightly better operator site for Hin repressor than hix $L$ is. Also, we found that by using the hix $C$ challenge phage, we could detect an apparent low level of repression even by Hin made from the chromosome.

Isolation and DNA sequence analysis of hix operator mutants defective in binding Hin

A strain carrying a P22 prophage that expresses $c 2$ repressor and a plasmid that expresses Hin (MS1582/ pKH66) is immune to superinfection by challenge phages with hixL, hix $R$, or hixC sites. In the absence of the Hin-producing plasmid, superinfecting challenge phages express antirepressor, inhibit $c 2$ repression, and form plaques efficiently. When challenge phages with hix sites are plated on a P22 lysogen that produces Hin, plaques that grow on the immune host arise at a low frequency $\left(10^{-5}\right)$. Phage from such plaques include mutants that carry hix operator mutations, which prevent repression by Hin protein and thereby permit the constitutive synthesis of antirepressor. 


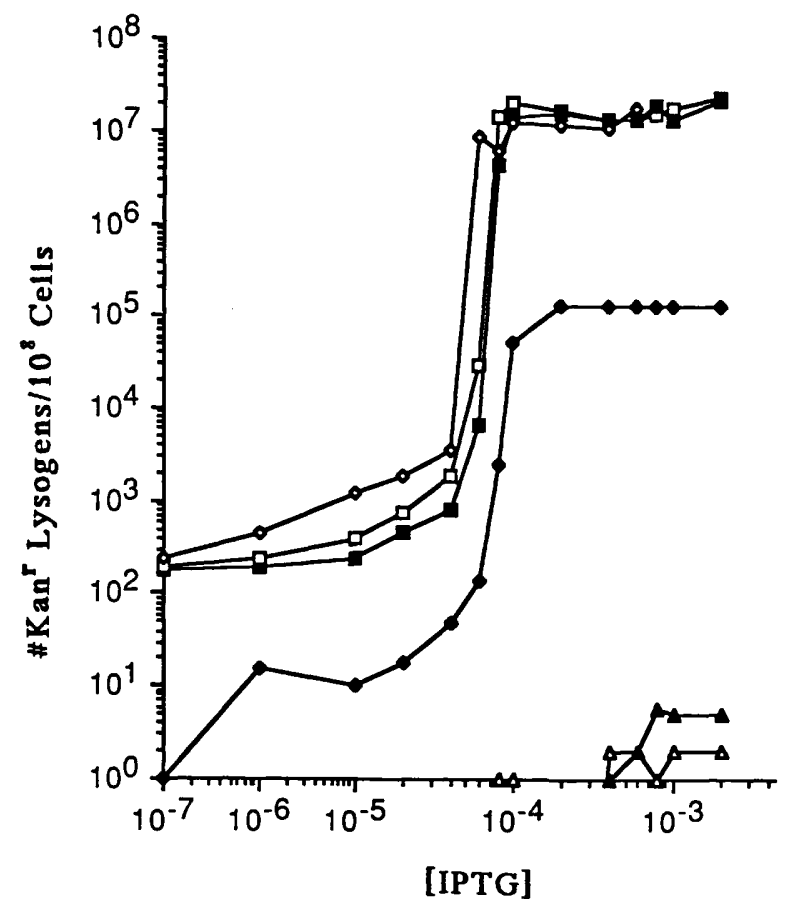

Figure 7. Challenge phage assays with the hix $L(N c o I)$ [orientations I ( $\square)$ and II $(-\mid), h i x C|\diamond|, h i x L+1(\mathrm{I}) \quad(\bullet \mid$, and hix $L+4(N c o I)[I(\Delta)$ and II $(\Delta)]$ challenge phages. The curves show frequency of lysogeny as a function of increasing IPTG levels (increasing Hin levels).

Independent plaque-forming mutants derived from hix $L$ challenge phages were isolated on such a host, and the DNA sequence of the hix region of each mutant phage was determined. Initially, we sequenced 33 independent spontaneous mutations in hixL. A majority of these (25) were deletions, 3 were single base pair insertions, and 1 was a substitution of a three $\mathrm{G}: \mathrm{C}$ base pair run for three $\mathrm{A}$ : $\mathrm{T}$ base pairs.

Although the deletion and insertion mutations we characterized were somewhat informative, we wanted to isolate single base pair substitution mutations to identify the particular DNA base pairs in the hix sequences that interact directly with the Hin protein in vivo. To enrich for single base pair substitution mutations, we irradiated hixL, hixR, or hixC phage with ultraviolet (UV) light prior to infection of an immune host that carried a plasmid (pGW1700) expressing the mucAB genes. The mucAB genes are derived from the conjugative Salmonella plasmid pKM101, and their expression increases the frequency of base pair substitutions in UVirradiated targets (Perry and Walker 1982).

The results of the analysis of mutant hix binding sites are shown in Figure 9. Only single base pair frameshift or substitution mutations are represented. Among mutant hix $R$ phages, all of the single base pair substitutions were found in the tighter binding half-site. We found three single base pair substitutions among 15 mutations sequenced in hixR. These include an A : T to G : C substitution at position 4 ; a change in a base shown to be important in minor groove contacts between Hin and hix $R$ in vitro (Sluka et al. 1987; A. Glasgow and M. Simon, unpubl.). Among $34 \mathrm{UV}$-induced hixL mutations, we found four different single base pair substitutions that change base pairs at positions 5 and 6 in both half-sites. Again, these four base pairs have been shown to be involved in minor groove contacts between Hin and hix $L$, based on the results of methylation protection and interference studies in vitro (A. Glasgow and $M$. Simon, unpubl.). Of 124 UV-induced mutations in the hix $C$ challenge phage, we recovered single base pair sub-

$\mathrm{immC}$

imml

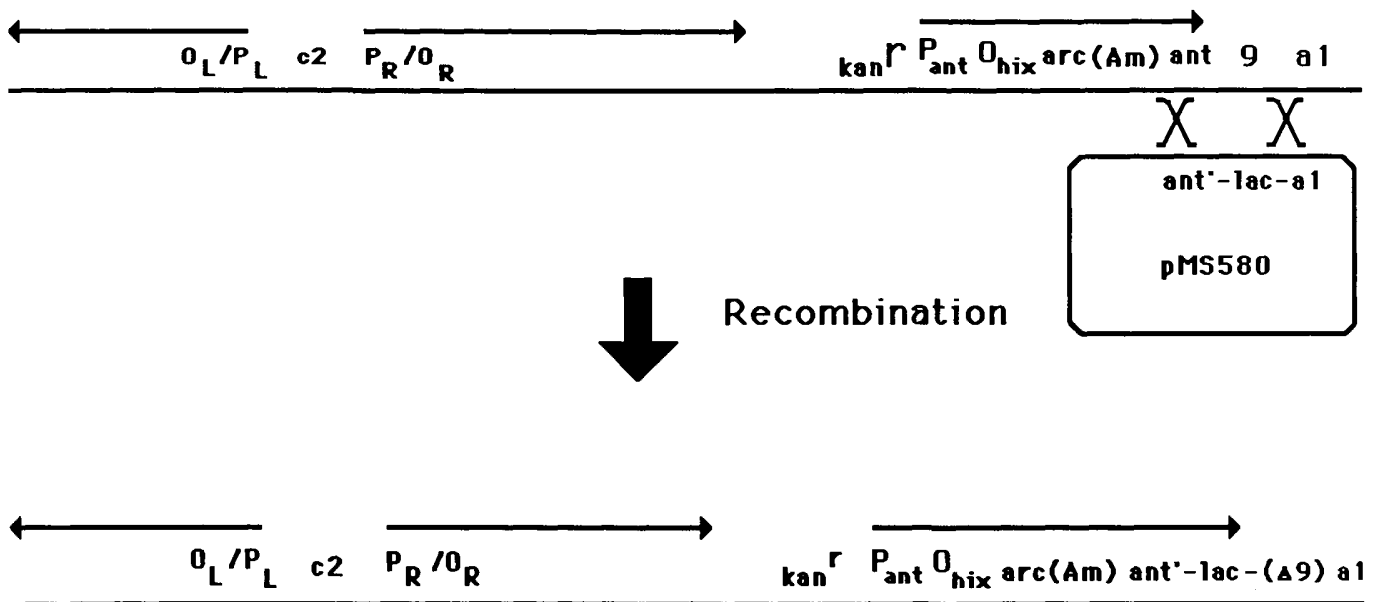

Figure 8. Construction of challenge phages with the lac operon under the control of the different hix operator sequences. Recombination between pMS580 and P22 phages at the ant and $a 1$ loci results in a phage carrying a fusion of the lac operon to the ant promoter. Recombinants are easily screened as blue plaques on X-gal-containing media. 
Table 1. $\beta$-Galactosidase activities of hix-lac fusion strains

\begin{tabular}{lc}
\hline Fusion & $\beta$-Galactosidase activity \\
\hline hix $L-l a c$ & 3000 \\
hix $L(+1)-l a c$ & 3300 \\
hix $L(N c o I)-l a c$ & 2500 \\
hix $L(+4)-l a c$ & 4600 \\
hixC-lac & 3500
\end{tabular}

a $\beta$-Galactosidase activity is expressed as nanomoles per minute per optical density unit (650 nm).

stitutions in the same four base pairs involved in minor groove contacts between Hin and the hix $L$ sequence. Only changes of $A: T$ to $G: C$ or $A: T$ to $C: G$ were obtained in these four base pairs.

\section{Conclusions}

To understand the Hin-mediated recombination reaction, we are attempting to study each of the individual steps involved in this ordered pathway of events. Here, we report the genetic characterization of the first step of the inversion reaction, the recognition and binding of Hin to the hix recombination sites in vivo. Hin recognizes and binds specific DNA segments. In addition to protein-DNA interactions, Hin is presumably involved in protein-protein interactions during the inversion reaction: (1) Hin forms multimers in the DNA-binding step of the reaction; (2) Hin multimers bound at the hixL and hixR sites may have a second interaction site required to form a larger multimer and bring the two different regions together; (3) Hin may also bind to the Fis protein as well, although Fis is not required to bring the two hix sites together (R.C. Johnson and M.I. Simon, unpubl.). Finally, Hin catalyzes a site-specific recombination reaction and releases itself from the inversion product.

To characterize the binding of Hin to the hix recombination sites in vivo, we have cloned hix sites at the startpoint of transcription of the phage P22 ant promoter and have examined the ability of Hin to bind the hix sites and repress the ant promoter. At first, it was unclear whether a recombinase would act as a repressor, i.e., whether or not Hin would bind tightly enough to a hix operator to repress transcription from the ant promoter. Nonetheless, we have found that Hin binds sufficiently to challenge phage DNA that includes either hix $L$ or hixR sites so that the challenge phage selection could be used to study Hin binding in vivo. Hin apparently represses better at the hix $L$ operator than at the hix $R$ operator. This phenotype enables us to study the binding of Hin to hix recombination sites in vivo and to separate binding from subsequent steps in the recombination reaction. These results are consistent with results found in the characterization of the in vitro properties of Hin binding to the hix sites (Sluka et al. 1987; A. Glasgow and M. Simon, unpubl.).

One of the unexpected results of this study is the finding that Hin will bind tighter to one of the three nat- ural hix half-sites, the one common to both hixL and $h i x R$. Hin binds less well to the unique hix $L$ and hixR half-sites (Fig. 4). This suggests that the Hin protein has a very high affinity for binding to the individual halfsites. The sequence of the common half-site that binds tightest to Hin was used to construct a perfectly symmetric hix site, hixC. The use of the hixC site will allow us to study the effects of symmetric changes in the hix sequence on binding to Hin.

The nature of the possible cooperative interaction of Hin with the hix sites was characterized by assaying the binding of Hin to hixL sites having either 1 or $4 \mathrm{bp}$ inserted between the hixL half-sites. The result of the hix $L+4$ challenge phage is consistent with multimeric binding of Hin to each half-site. We anticipated that the 4-bp insertion would rotate the half-sites to different faces of the DNA helix and thereby greatly impair multimeric binding. As expected, insertion of 4 bp between the hixL half-sites eliminates multimeric binding, as measured by the challenge selection. Hin-mediated repression of the hixL +4 phage is not as great as that with the hixC half-site alone. This is probably due to competition between the two rotated half-sites in the $h i x L+4$ phage in binding to Hin, thereby reducing overall binding to the promoter-proximal half-site necessary for repression to occur.

The hix $L+1$ site, with a single base-pair insertion, appears defective in binding. The single base-pair insertion does not interfere with the sigmoidal lysogenization curve, but the complex formed between the mutant hix $L+1$ site and Hin appears not to be as stable as with the hix $L$ site, because repression and therefore apparent binding of the hixL +1 phage are down at all IPTG concentrations. If cooperative binding was reflected by the sigmoidal lysogenization curve, the low lysogenization frequencies at low Hin concentrations would represent monomer binding. If this were due to cooperative binding of monomers, the hix $L+1$ challenge phage should not exhibit reduced lysogenization frequencies at low IPTG levels as compared with hixL. The fact that it does suggests that Hin binds to the hix sites as a multimer at all IPTG levels and the sigmoidal lysogenization curve is an intrinsic property of repression of the ant promoter and does not reflect cooperative binding of monomers.

The repression of ant by the binding of Hin to hix $R$ was found to be orientation dependent (Fig. 4). Hin represses when hix $R$ is in the orientation that places the tighter binding half-site proximal to the startpoint of ant transcription better than when the poorer half-site is promoter proximal. One explanation consistent with this result is that it reflects a difference in the competition between RNA polymerase and Hin for binding to the promoter-operator region in these two situations. When the hix $R$ site is oriented with respect to the ant promoter so that the poorer hixR half-site is promoter proximal, a higher concentration of Hin is required to repress transcription. It may be that when the weaker binding half-site of hixR is promoter proximal, RNA polymerase can outcompete (or essentially 'peel off') the 

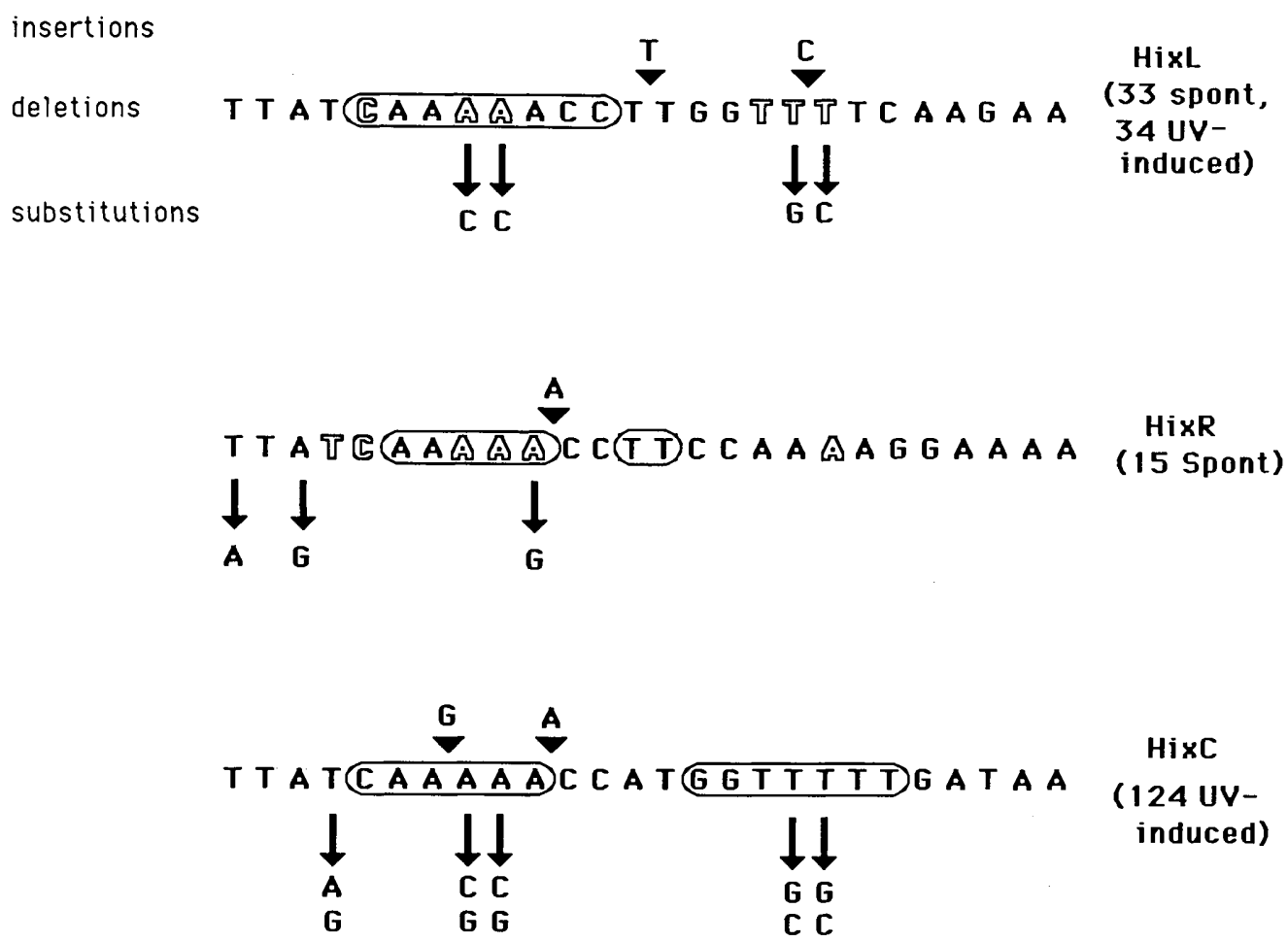

Figure 9. Single base changes in hixL, hixR, and hix $C$ which reduce binding to Hin. The circled bases are those that are reduced for binding Hin when singly deleted. The outlined bases in hixL and hix $R$ are those base pairs that prevent Hin binding in vitro when methylated (A. Glasgow and M. Simon, unpubl.).

bound Hin multimer at the lower Hin concentrations.

The binding of Hin to the hixL half-sites is also orientation dependent. The dependence of the frequency of lysogeny of a challenge phage on the orientation of a cloned half-site may not reflect a difference in Hin binding; rather, the correct position of the bound Hin repressor with respect to the ant promoter could be a critical determinant of the ability of Hin to repress transcription. The results of in vitro experiments suggest that Hin binds the minor groove in the center of the hix dyad and the major groove at the outside regions (A. Glasgow and $M$. Simon, unpubl.). Thus, a full site would bind the same faces of the DNA relative to the ant promoter regardless of orientation. In contrast, Hin would bind the half-site in the major groove in the promoterproximal portion in one orientation and the minor groove in the promoter-proximal portion of the hix halfsite in the other orientation. Thus, the orientation of the half-site would determine which side of the DNA helix bound relative to the ant promoter, which may greatly affect the ability of Hin to repress transcription.

The binding of Hin to hix sites in vivo is tight enough to construct a host immune to infection by the hixL, hixR, and hix $C$ challenge phages. Such a host carries a P22 $c 2+$ prophage and a plasmid expressing Hin. The hix $L$, hixR, and hixC challenge phages cannot form plaques on this strain, because Hin is able to repress ant transcription. On the basis of this phenotype, we have selected mutants that result from DNA base changes in hix sites that impair Hin binding. Rare plaques that arise when hix challenge phages are plated on a 'Hin-immune' host carry mutations in their hix sites. More than 200 hix operator-constitutive mutations were sequenced to determine which base pairs in the hix sites are essential for Hin binding in vivo. Sequence analysis of hix $L$ and hix $C$ mutations shows that the $\mathrm{A}$ : $\mathrm{T}$ base pairs at positions 5, 6, and 10 in the hix site are critical for binding. These results are consistent with studies showing that A : T base pairs at positions 4,5 , and 6 prevent Hin binding in vitro when methylated in the minor groove, (A. Glasgow and M. Simon, unpubl.). The C : G base pair at position 9 also prevents Hin binding when methylated in the major groove.

We did not recover $\mathrm{A}: \mathrm{T}$ to $\mathrm{T}: \mathrm{A}$ transversion mutations at positions 5 and 6 of the hix site. Hin binds both hix L, which has A : T base pairs at positions 5 and 6 and $T: A$ base pairs at positions -5 and -6 , and hix $R$ which has $\mathrm{A}$ : $\mathrm{T}$ base pairs in both half-sites. Hin may contact these runs of $\mathrm{A}: \mathrm{T}$ base pairs in the minor groove at positions 5 and 6 . All of the mutations in hix $R$ that impair binding to Hin were found in the tighter binding halfsite. In addition, three base substitution mutations were obtained that were not observed among the 150 hix $L$ and hix $C$ mutants sequenced. This may suggest that the hix $R$ sequence is more sensitive to base substitution mutation, because one hix $R$ half-site is defective in 
binding when compared to the other half-site. We presume that weaker contact points between Hin and hix were identified by mutations in hixR that were not found in hixL or hixC.

The results presented here demonstrate the utility of the challenge phage selection in the characterization of the in vivo binding properties of Hin recombinase to its recombination sites. A second advantage of using Hin to repress ant transcription in bacteriophage P22 is the ability to construct an immune host allowing for the direct selection of hix site mutants defective in binding to Hin. We hope to use these mutants to select for Hin suppressor mutants that can regain the ability to bind to mutant hix sites and allow the mutant challenge phages to lysogenize. Such studies will enable us to determine the specific amino acids in Hin that make specific base contacts in the hix sites.

\section{Materials and methods}

\section{Bacteria and bacteriophage strains}

All strains used in this study and their sources are listed in Table 2. All S. typhimurium strains are derived from LT2 strain DB7000 (Susskind 1980). E. coli strains are derived from K-12. P22 Kn9 arcH1605 (am), the parent of the hix-challenge phages, has been described (Youderian et al. 1983). The hix-challenge phages were constructed by crosses between $\mathrm{P} 22 \mathrm{Kn} 9$ arcH1605 and plasmids in which various hix sequences had been cloned in place of the ant operator (Fig. 3).

\section{Plasmids}

Plasmids used in this study and their sources are listed in Table 1. The construction of plasmids carrying hix DNA sequences at the P22 ant operator locus (Fig. 3) was done essentially as described for other operator sequences (Benson et al. 1986). A linker tailing protocol was used to clone synthetic hix sites (double-stranded oligonucleotides) into the $S \mathrm{maI}$ site of plasmid (Bass et al. 1987). Reaction mixtures contained $1 \mu$ l SmaI-digested pPY 190 DNA $(1 \mathrm{mg} / \mathrm{ml}), 6.5 \mu$ l hybridized unphosphorylated oligonucleotides $(1-10 \mathrm{mg} / \mathrm{ml}), 1.5 \mu \mathrm{l} 10 \times$ DNA ligase/ kinase buffer $\left[0.66 \mathrm{~m}\right.$ Tris- $\mathrm{HCl}(\mathrm{pH} 7.5), 100 \mathrm{~mm} \mathrm{MgCl}_{2}, 2$ $\mathrm{mg} / \mathrm{ml}$ bovine serum albumin], $3 \mu \mathrm{l} 10 \mathrm{mM}$ ATP, $3 \mu \mathrm{l} 100 \mathrm{~mm}$ dithiothreitol, and $1.5 \mu \mathrm{l}$ of T4 DNA ligase (New England Biolabs). Ligations were carried out overnight at room temperature. After ligation, $5 \mu \mathrm{l}$ of $3 \mathrm{~m}$ sodium acetate $(\mathrm{pH} 5)$ and $80 \mu \mathrm{l}$ of cold $\left(-20^{\circ} \mathrm{C}\right)$ ethanol were added. The solutions were mixed and stored at $-70^{\circ} \mathrm{C}$ for $10 \mathrm{~min}$. The DNA/protein mixtures were pelleted in a microfuge $\left(15 \mathrm{~min}\right.$ at $\left.4^{\circ} \mathrm{C}\right)$, rinsed with $70 \%$ ethanol, $30 \% 10 \mathrm{~mm}$ Tris- $\mathrm{HCl}(\mathrm{pH} 7.5)$, and $0.1 \mathrm{~mm}$ EDTA and vacuum-dried. Dried pellets were resuspended in $20 \mu \mathrm{l}$ of 10 $\mathrm{mm}$ Tris- $\mathrm{HCl}(\mathrm{pH} 7.5)$ and incubated at $65^{\circ} \mathrm{C}$ for $5 \mathrm{~min}$. A $66-\mu \mathrm{l}$ aliquot of $\mathrm{H}$ buffer $[100 \mathrm{~mm} \mathrm{NaCl}, 10 \mathrm{~mm}$ Tris- $\mathrm{HCl}(\mathrm{pH}$ 7.5), 5 mM EDTA] was added to each mixture, the mixtures were incubated at $65^{\circ} \mathrm{C}$ for $10 \mathrm{~min}$, set at room temperature for $10 \mathrm{~min}$, and then put on ice or frozen for later use. A $20-\mu \mathrm{l}$ aliquot of each solution was used to transform $200 \mu \mathrm{l}$ of frozen and thawed competent DHl cells. Plasmids carried by ampicillinresistant $\left(\mathrm{Amp}^{\mathrm{x}}\right)$ transformants were screened for insertions of the synthetic hix sites by digestion of plasmid DNA with EcoRI, followed by $5 \%$ polyacrylamide gel electrophoresis. The pPY190 plasmid has 341-bp sequence that includes the SmaI cloning site. Plasmids that had a larger EcoRI fragment than the parent were kept as putative inserts.
The source of Hin used in this study is the moderate copy number plasmid PKH66, which expresses the hin gene from the tac promoter, under the control of the $1 a c I^{\mathrm{Q}}$ gene. The original $\mathrm{P}_{\text {tac }}-$ hin construct was on a high-copy-number plasmid pMS571 (Johnson and Simon 1985). In strains carrying pMS571, levels of IPTG $>10^{-5} \mathrm{M}$ were lethal to cell growth when plasmid selection was maintained. At high IPTG concentrations, the Hin protein aggregated into insoluble inclusion bodies. The accumulation of large amounts of these insoluble inclusion bodies may be the cause of lethality in cells where Hin is highly overproduced. In strains carrying pKH66, IPTG levels as high as $2 \mathrm{~mm}$ have no noticeable effects on cell growth. Thus, pKH66 provides a source of Hin that is well repressed by the $L a c I^{\circ}$ gene product but can be varied in response to the concentration of IPTG present in the growth medium. The pKH66 plasmid is present in low copy and presumably produces much less Hin when fully induced than from the pMS571 plasmid. The plasmid PKH66 was constructed by cloning the $\mathrm{P}_{\text {tac }}$-hin fragment of plasmid PMS571 into the BamHI site of plasmid pMS421. The pMS571 plasmid was partially digested with $\mathrm{BamHI}$ and electrophoresed on a $1 \%$ agarose gel. The 1.1$\mathrm{kb}$ fragment was purified and ligated into BamHI-digested plasmid pMS421, resulting in plasmid pKH66.

\section{Media}

The E medium of Vogel and Bonner (Vogel and Bonner 1956), supplemented with $0.2 \%$ dextrose, was used as minimal medium. Alternative carbon sources were supplemented to $0.2 \%$ in E medium lacking citrate (Maloy and Roth 1983). Luria-Bertani (LB) medium (Difco Tryptone, $10 \mathrm{~g} /$ liter; Difco yeast extract, $5 \mathrm{~g} /$ liter; $\mathrm{NaCl}, 5 \mathrm{~g} / \mathrm{liter}$ ) was used as rich medium. Auxotrophic supplements were included in media at final concentrations described previously (Davis et al. 1980). Antibiotics (Sigma) were included in media as needed (final concentrations given]: ampicillin [100 $\mu \mathrm{g} / \mathrm{ml}$ for plasmid selection, $30 \mu \mathrm{g} / \mathrm{ml}$ for Mud lysogen selection (Hughes and Roth 1984)], chloramphenicol $(12.5 \mu \mathrm{l} / \mathrm{ml})$, kanamycin sulfate $(40 \mu \mathrm{l} / \mathrm{ml})$, spectinomycin $\left(\mathrm{Spc}_{1} 100 \mu \mathrm{l} / \mathrm{ml}\right)$, streptomycin $[\mathrm{Str} ; 50 \mu \mathrm{l} / \mathrm{ml}$ for plasmid selection, $1 \mathrm{mg} / \mathrm{ml}$ for chromosomal $\operatorname{rps} L$ (strA) mutant selection], and tetracycline $(10 \mu \mathrm{l} / \mathrm{ml})$. Bacterial cell growth was monitored at $540 \mathrm{~nm}$ in a Klett-Summerson colorimeter.

\section{Transductional methods}

For all transductional crosses, the high-frequency generalized transducing mutant of bacteriophage P22 (HT105/1 int-201) was used (Sanderson and Roth 1983). Selective plates were spread with $2 \times 10^{8}$ to $2 \times 10^{9}$ phage. Transductants were purified as described (Hughes and Roth 1985). For antibiotic selections other than ampicillin or tetracycline, phage and cells were mixed and left at room temperature for $1 \mathrm{hr}$ prior to spreading on selective plates to permit expression of the antibiotic-resistance phenotype.

\section{Challenge phage assay}

An overnight culture of strain MS1868 carrying the Hin-producing plasmid pKH66 was diluted 100-fold into LB plus Str and Spc to maintain plasmid selection and was grown to a density of $100 \mathrm{Klett}$ units $\left(\sim 6 \times 10^{8}\right.$ cells $\left./ \mathrm{ml}\right)$. Cells were diluted fourfold into the same medium plus varying amounts of IPTG and grown for $1 \mathrm{hr}$ to permit the induction of Hin expression from plasmid pKH66. P22 hix challenge phage was added to a m.o.i. of 20 , and infected cells were incubated for $1 \mathrm{hr}$ at room temperature to allow the expression of the $\operatorname{Kan}^{\mathrm{r}}$ phenotype. 
Table 2. List of strains and plasmids

\begin{tabular}{|c|c|c|c|}
\hline \multicolumn{3}{|l|}{ Strains } & Source ${ }^{\mathbf{a}}$ \\
\hline \multicolumn{4}{|c|}{ E. coli } \\
\hline \multirow{2}{*}{\multicolumn{3}{|c|}{$\begin{array}{l}\mathrm{DH} 1: \mathrm{F}^{-} \text {thi-1 supE44 hsdR17 endA1 recA1 relA1 gyr-96 } \lambda^{-} \\
\text {MC1000:F- thi araD139 } \Delta\left(\text { araBAOIC-leu } 7679 \text { galU galK lacY74 rpsL } \lambda^{-}\right.\end{array}$}} & D. Hanahan \\
\hline & & & M. Casadaban \\
\hline \multicolumn{4}{|c|}{ S. typhimurium } \\
\hline \multicolumn{4}{|c|}{ MS1362:leuA414(Am) supD Fels- } \\
\hline \multicolumn{4}{|c|}{ MS1363:leuA414 supE40 Fels- } \\
\hline \multicolumn{4}{|c|}{ MS1367:leuA414 supE40 Fels- ataA::[P22 sieA44 Ap2 DEL7283 (immI) (Ap $\left.\left.{ }^{\mathrm{r}}\right)\right]$} \\
\hline \multicolumn{4}{|c|}{ MS1582:leuA414 supE40 Fels- ataA::[P22 sieA44 16-amH1455 tpfr-49] } \\
\hline \multicolumn{4}{|c|}{ MS1868:leuA414 hsdSB Fels ${ }^{-}$} \\
\hline \multicolumn{4}{|c|}{ MS1882:leuA414 hsdSB endA Fels ${ }^{-}$} \\
\hline \multicolumn{4}{|c|}{ MS1883:leuA414 hsdSB supE40 Fels- } \\
\hline \multicolumn{4}{|c|}{ MS2544:1euA414 supE40 hsdSB recA1 Fels- } \\
\hline \multicolumn{4}{|c|}{$\begin{array}{l}\text { SL4213:hsdL6 hsdSA29 galE496 metA22 metE55 ilv-452 rpsL120 xyl-404 H1-b H2-e,n,x nml- Fels }{ }^{-} \\
\left.\text {TH564:leuA414 supE40 Fels }{ }^{-} \text {ataA::[P22 sieA44 Ap7 tpfr184 } \Delta(m n t-a 1)\left(\mathrm{Ap}^{\mathrm{s}}\right)\right]\end{array}$} \\
\hline \multicolumn{2}{|l|}{ Plasmids } & Source & \\
\hline pMS571 & $\left(\mathrm{P}_{t a c}-\mathrm{Hin}^{+}, \mathrm{Ap}^{\mathrm{r}}\right)$ & Johnson and Simon (1985) & \\
\hline pMS421 & [pSC101 origin (low copy no.), lacI $\mathrm{Q}, \mathrm{Str}^{\mathrm{r}}, \mathrm{Spc}^{\mathrm{r}}$ ] & M. Susskind & \\
\hline pKH66 & $\left(\mathrm{pMS} 421 \mathrm{P}_{t a c}-\mathrm{Hin}^{+}\right)$ & this paper & \\
\hline pPY190 & (pBR322, Pant-arc-ant') & P. Youderian & \\
\hline pMS580 & (pBR322, Pant-lacZYA'- $\Delta 9$ al) & M. Susskind & \\
\hline
\end{tabular}

a Unless indicated otherwise, all strains originated from M. Susskind's lab.

After $1 \mathrm{hr}$, dilutions of the adsorption mixture were plated on LB-Kan plates containing the same concentration of IPTG used for induction and incubated overnight at $37^{\circ} \mathrm{C}$. As a negative control, strain MS1868 carrying plasmid pMS421, which is otherwise isogenic with pKH66 but lacks the Hin gene, was used. None of the hix-challenge phages were able to lysogenize this strain.

\section{DNA sequence analysis}

Plasmid DNA and double-stranded phage DNA were sequenced by the method of Sanger et al. (1977).

\section{Western blot analysis of Hin levels}

The ProtoBlot Immunoscreening System protocol (Promega) was used to assay Hin levels expressed from plasmid pKH66 under different induction conditions. Strain MS1883 with plasmid pKH66 was grown overnight at $37^{\circ} \mathrm{C}$ in $\mathrm{LB}$ medium containing Str and Spc to maintain plasmid selection. The culture was diluted 100-fold in LB plus Str and Spc and grown to O.D. 0.8 and then diluted 4-fold into LB plus Str and Spc and the following concentrations of IPTG (final molar concentration given): $2 \times 10^{-5}, 4 \times 10^{-5}, 6 \times 10^{-5}, 8 \times 10^{-5}$, $8 \times 10^{-4}, 2 \times 10^{-4}, 4 \times 10^{-4}, 6 \times 10^{-4}$. The cultures were grown for an additional hour at $37^{\circ} \mathrm{C}$ to allow for induction of Hin (final O.D. 5-6). Cells (10 ml) from each IPTG concentration used were pelleted by centrifugation and resuspended in 5 $\mathrm{ml}$ of LB. A unit sample of O.D. 1 of each culture was electrophoresed in a $15 \%$ SDS-polyacrylamide (4\% stacking) gel and hybridized with Hin antibody prepared previously (Bruist and Simon 1984). Purified Hin (Johnson et al. 1986) was also run as a standard.

\section{Acknowledgments}

We thank Carol Lee for help with the DNA sequence and Western blot analysis; Steve Bass, Nicholas Benson, Brigitte Lebreton, Paul Sugiono, and Miriam Susskind for helpful discus- sions; and Anna Glasgow and Robert Bourret for critically reading this manuscript. This work was supported by grants from the National Science Foundation and from the Defense Advanced Research Projects Agency (DARPA) Institutional Research Initiative Program to M.I.S. and grant GM34982 from the National Institutes of Health to P.Y. K.T.H. was supported by fellowship GM11088 from the National Institutes of Health.

\section{References}

Bass, S., P. Sugiono, D.N. Arvidson, R.P. Gunsalas, and P. Youderian. 1987. DNA specificity determinants of Escherichia coli tryptophan repressor binding. Genes Dev. 1: 565-572.

Benson, N., P. Sugiono, and P. Youderian. 1987. DNA sequence determinants of $\lambda$ repressor binding in vivo. Genetics 118: $21-29$.

Benson, N., P. Sugiono, S. Bass, L.V. Mendelman, and P. Youderian. 1986. General selection for specific DNA-binding activities. Genetics 114: 1-14.

Bruist, M.F. and M.I. Simon. 1984. Phase variation and Hin protein: In vitro activity measurements, protein overproduction, and purification. J. Bacteriol. 159: 71-79.

Bruist, M.F., A.C. Glasgow, R.C. Johnson, and M.I. Simon. $1987 \mathrm{a}$. Fis binding to the recombinational enhancer of the Hin DNA inversion system. Genes Dev. 1: 762-772.

Bruist, M.F., S.J. Horvath, L.E. Hood, T.A. Steitz, and M.I. Simon. 1987b. Synthesis of a site-specific DNA-binding peptide. Science 235: 777-780.

Davis, R.W., D. Botstein, and J.R. Roth. 1980. Advanced bacterial genetics. Cold Spring Harbor Laboratory, Cold Spring Harbor, New York.

Huber, H.E., S. Iida, W. Arber, and T.A. Bickle. 1985. Site-specific DNA inversion is enhanced by a DNA sequence in cis. Proc. Natl. Acad. Sci. 82: 3776-3780.

Hughes, K.T. and J.R. Roth. 1984. Conditionally transpositiondefective derivative of $\mathrm{Mu} \mathrm{d} 1$ (Amp Lac). J. Bacteriol. 159: 130-137.

- 1985. Directed formation of deletions and duplications using mud(Ap, lac). Genetics 109: 263-282. 
Iino, T. 1969. Genetics and chemistry of bacterial flagella. Bacteriol. Rev. 33: 454-475.

1977. Genetics of structure and function of bacterial flagella. Annu. Rev. Genet. 11: 161-182.

Johnson, R.C. and M.I. Simon. 1985. Hin-mediated site-specific recombination requires two $26 \mathrm{bp}$ recombination sites and a 60 bp recombinational enhancer. Cell 41: 781-791.

Johnson, R.C., M.F. Bruist, and M.I. Simon. 1986. Host protein requirements for in vitro site-specific DNA inversion. Cell 46: $531-539$.

Johnson, R.C., A.C. Glasgow, and M.I. Simon. 1987. Spatial relationship of the Fis binding sites for Hin recombinational enhancer. Nature 329: 462-465.

Kahman, R., F. Rudt, C. Koch, and G. Mertens. 1985. G inversion in bacteriophage Mu DNA is stimulated by a site within the invertase gene and a host factor. Cell 41: 771780.

Kahman, R., G. Mertens, A. Klippel, B. Brauer, F. Rudt, and C. Koch. 1987. The mechanism of $G$ inversion. In DNA replication and recombination, pp. 681-690. Alan R. Liss, New York.

Kutsukake, K. and T. lino. 1980. Inversions of specific DNA segments in flagellar phase variation of Salmonella and inversion systems of bacteriophages $\mathrm{P} 1$ and $\mathrm{Mu}$. Proc. Natl. Acad. Sci. 77: 7238-7341.

Lebreton, B., P. Youderian, P.V. Prasad, and M. Jayaram. 1988. Mutations that improve the binding of yeast FLP recombinase to its substrate. Genetics 118: $393-400$.

Maloy, S.R. and J.R. Roth. 1983. Regulation of proline utilization in Salmonella typhimurium: Characterization of put :: $\mathrm{Mu} d(\mathrm{Ap}$, lac $)$ operon fusions. J. Bacteriol. 154: 561568.

Perry, K.L. and G.C. Walker. 1982. Identification of plasmid (pKM101)-coded proteins involved in mutagenesis and UV resistance. Nature 300: 278-281.

Plasterk, R.H.A., A. Brinkman, and P. van de Putte. 1983. DNA inversions in the chromosome of $E$. coli and in bacteriophage Mu: Relationship to other site-specific recombination systems. Proc. Natl. Acad. Sci. 80: 5355-5358.

Sanderson, K.E. and J.R. Roth. 1983. Linkage map of Salmonella typhimurium. Microbiol. Rev. 47: 410-453.

Sanger, F., S. Nicklen, and A. Coulson. 1977. DNA sequencing with chain terminating inhibitors. Proc. Natl. Acad. Sci. 74: 5463-5476.

Silverman, M. and M. Simon. 1980. Phase variation: Genetic analysis of switching mutants. Cell 19: 845-854.

Silverman, M., J. Zieg, and M.I. Simon. 1979. Flagellar-phase variation: Isolation of the $\mathrm{rH1}$ gene. J. Bacteriol. 137: 517523.

Sluka, J.P., S.J. Horvath, M.F. Bruist, M.I. Simon, and P.B. Dervan. 1987. Synthesis of a sequence-specific DNAcleaving peptide. Science 238: 1129-1132.

Stocker, B.A.D. 1949. Measurements as rate of mutation of flagellar antigenic phase in Salmonella typhimurium $J$. Hygiene 47: 398-413.

Susskind, M.M. 1980. A new gene of bacteriophage P22 which regulates synthesis of antirepressor. I. Mol. Biol. 138: 685713.

Susskind, M.M. and P. Youderian. 1983. Bacteriophage P22 antirepressor and its control. In Lambda II led. R.W. Hendrix, J.W. Roberts, F.W. Stahl, and R.A. Weisberg), pp. 347-363. Cold Spring Harbor Laboratory, Cold Spring Harbor, New York.

Szekely, E. and M. Simon. 1983. DNA sequence adjacent to flagellar genes and evolution of flagellar-phase variation. $I$. Bacteriol. 155: 74-81.
Vogel, H.J. and D.M. Bonner. 1956. Acetylornithase of Escherichia coli: Partial purification and some properties. J. Biol. Chem. 318: 97-106.

Youderian, P., A. Vershon, S. Bouvier, R.T. Sauer, and M.M. Susskind. 1983. Changing the DNA binding specificity of a repressor. Cell 35: 777-783.

Zieg, J. and M. Simon. 1980. Analysis of the nucleotide sequence of an invertible segment of an invertible controlling element. Proc. Natl. Acad. Sci. 77: 4196-4200.

Zieg, J., M. Hilmen, and M. Simon. 1978. Regulation of gene expression by site-specific inversion. Cell 15: 237-244.

Zieg, J., M. Silverman, M. Hilmen, and M. Simon. 1977. Recombinational switch for gene expression. Science 196: $170-172$. 


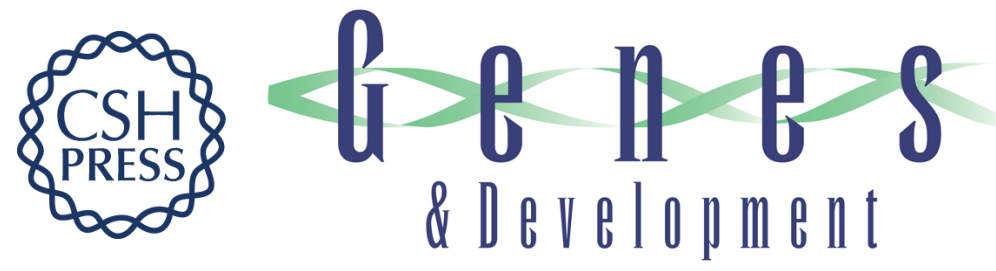

\section{Phase variation in Salmonella: analysis of Hin recombinase and hix recombination site interaction in vivo.}

K T Hughes, $\mathrm{P}$ Youderian and M I Simon

Genes Dev. 1988, 2:

Access the most recent version at doi:10.1101/gad.2.8.937

References This article cites 33 articles, 19 of which can be accessed free at:

http://genesdev.cshlp.org/content/2/8/937.full.html\#ref-list-1

License

Email Alerting

Service

Receive free email alerts when new articles cite this article - sign up in the box at the top right corner of the article or click here.

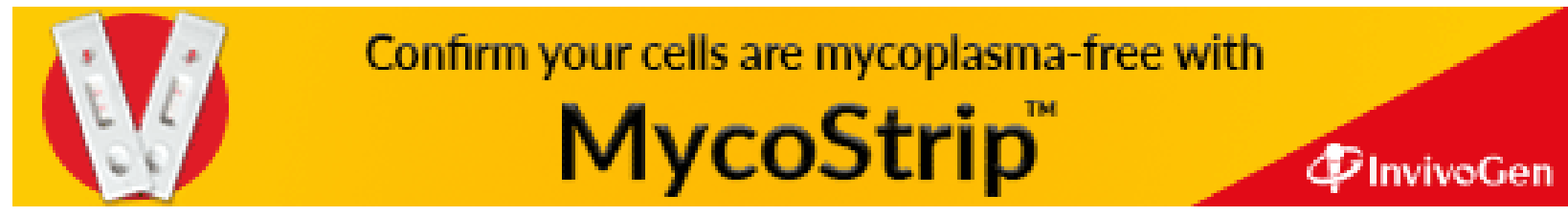

